\title{
Comparison of Urine Flow Cytometry on the UF-1000i System and Urine Culture of Urine Samples from Urological Patients
}

\author{
Moritz Fritzenwanker ${ }^{a} \quad$ Marcel Oliver Grabitz ${ }^{a} \quad$ Borros Arneth $^{b} \quad$ Harald Renz $^{b}$ \\ Can Imirzalioglu ${ }^{a}$ Trinad Chakraborty ${ }^{a}$ Florian Wagenlehner ${ }^{c}$

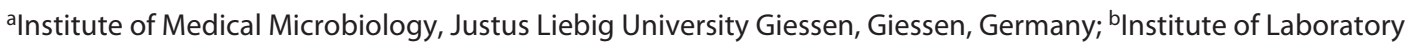 \\ Medicine and Pathobiochemistry, Phillips University Marburg, Marburg, Germany; ${ }^{\circ}$ Clinic of Urology, Pediatric \\ Urology and Andrology, Justus Liebig University Giessen, Giessen, Germany
}

\section{Keywords}

Urine flow cytometry · Fast urine diagnostic · Infection control · Presurgical screening • Urinary tract infections . Bacteriuria

\begin{abstract}
Introduction: The aims of this study were to evaluate urine flow cytometry (UFC) as a tool to screen urine samples of urological patients for bacteriuria and to compare UFC and dipstick analysis with urine culture in a patient cohort at a urological department of a university hospital. Methods and Material: We screened 662 urine samples from urological patients (75.2\% male; $80.7 \%$ inpatients; mean age 58 years). UFC results were compared to microbiological urine culture. Results: The accuracy in using the UFC-based parameters for detecting cultural bacteriuria was $91.99 \%$ and $88.97 \%$ for $\geq 10^{5}$ colony-forming units (CFU) $/ \mathrm{mL}$ and $\geq 10^{4} \mathrm{CFU} / \mathrm{mL}$, respectively. UFC and leukocyte dipstick analysis measured leukocyturia similarly (Pearson correlation coefficient $0.87, p$ value $<0.01 \%$ ), but dipstick analysis scored less accurately on bacteriuria (accuracy $59.37 \%$ and $62.69 \%$ ). UFC remained effective in subgroup analysis of patients of both sexes and with different urological conditions with its overall use only slightly impaired when assessing gross hematuria (NPV $84.62 \%$ for $\geq 10^{4} \mathrm{CFU} / \mathrm{mL}$ ). UFC also reliably removed those
\end{abstract}

urine samples below cutoffs with negative predictive values of $99.28 \%$ for $\geq 10^{5} \mathrm{CFU} / \mathrm{mL}$ and $95.86 \%$ for $\geq 10^{4} \mathrm{CFU} / \mathrm{mL}$. Conclusion: Counting bacteria with UFC is an accurate and rapid method to determine significant bacteriuria in urological patients and is superior to dipstick analysis or indirect surrogate parameters such as leukocyturia. When UFC is available, we recommend it to be used for the diagnosis of bacteriuria over findings obtained by dipstick analysis.

(c) 2021 The Author(s).

Published by S. Karger AG, Basel

\section{Introduction}

Urinary tract infections (UTIs) are among the most common bacterial infections. The gold standard for laboratory diagnosis is classical urine culture [1], which usually takes at least $24 \mathrm{~h}$ and $48 \mathrm{~h}$ when including resistance testing. Initial antibiotic therapy is therefore most often empirically calculated. International guidelines recommend usage of antibiotics like nitrofurantoin and fosfomycin for uncomplicated UTI (due to low resistance rates and resistance development). Antibiotics may be prescribed on signs and symptoms alone, when bacteria are

Moritz Fritzenwanker, Marcel Oliver Grabitz, and Borros Arneth contributed equally to this work.
(C) 2021 The Author(s)

Published by S. Karger AG, Basel

This is an Open Access article licensed under the Creative Commons Attribution-NonCommercial-4.0 International License (CC BY-NC) (http://www.karger.com/Services/OpenAccessLicense), applicable to the online version of the article only. Usage and distribution for commercial purposes requires written permission.
Correspondence to:

Moritz Fritzenwanker, moritz.fritzenwanker@ mikrobio.med.uni-giessen.de 
not even present; therefore, rapid diagnostic methods to diagnose bacteriuria or its absence are an important step to promote unnecessary usage of antibiotics [2].For point-of-care results, urine is often tested using strips with leukocyte esterase and nitrite, that is, "dipsticks," to indirectly diagnose UTI. The reliability of this approach is an ongoing subject of discussion [3]. In recent years, urine flow cytometry (UFC) has been evaluated as a reliable and efficient method to diagnose bacteriuria, revealing heterogeneous results in different patient populations [4-6]. Patients scheduled for surgery are of particular interest, as asymptomatic bacteriuria is of relevance in urothelium-traumatizing urological procedures [1].

Current guidelines generally recommend screening for bacteriuria prior to urological interventions but concur in the assessment that reagent strip analysis is not accurate enough to exclude bacteriuria and thus recommend against using it as the sole method of screening [1]. Similarly, data on UFC is deemed insufficient as it has not been studied in the context of urological patient populations. Our study aimed to evaluate UFC as a tool to screen urine samples, particularly in connection with those patients undergoing urological examination. We compare the data obtained by UFC to dipstick analysis as well as to classical methods of urine culture.

\section{Materials and Methods}

\section{Urine Samples}

We analyzed consecutive urine samples from patients of the urological department of a university hospital with the UF-1000i system (Sysmex, Kobe, Japan).

\section{UF-1000i}

The UF-1000i system is a flow cytometer that combines impedance method detection with the identification and quantification of urinary particles by flow cytometry of the urine. UF-1000i has the advantage of having a separate bacterial analysis channel. It analyzes 65,000 particles per sample within 50-75 s and has a throughput of 90-100 urine samples per hour. In the special highprecision bacterial mode, the maximum throughput is up to 80 samples per hour and requires either a minimum volume of $1.0 \mathrm{~mL}$ of centrifuged or uncentrifuged native urine in the manual mode or $4 \mathrm{~mL}$ in the automated sampler mode, which was used here. Consequently, it is appropriate for high-throughput screening of urine samples for UTIs. The default parameters for classifying UTIs, which are often defined as $>20 \mathrm{WBCs} / \mu \mathrm{L}$, are not suitable for practical diagnosis in many cases.

The UF-1000i analyzer can discriminate and count 12 diagnostic parameters of cells and formed elements and 2 calculated parameters. These parameters are white blood cells, red blood cells, epithelial cells, casts, bacteria, small round cells, yeast-like cells, spermatocytes, crystals, pathological casts, mucus, and conductivity. The system employs fluorescence flow cytometry technology,
Table 1. Patient characteristics

\begin{tabular}{|c|c|c|c|}
\hline All samples & 662 & & \\
\hline \multicolumn{4}{|l|}{ Sex, $n(\%)$} \\
\hline Male & $498(75.2)$ & & \\
\hline Female & $164(24.8)$ & & \\
\hline \multicolumn{4}{|l|}{ Age, years } \\
\hline Mean \pm SD & $58.25 \pm 17.21$ & & \\
\hline \multicolumn{4}{|c|}{ Hospital admission, $n(\%)$} \\
\hline Inpatient & $534(80.7)$ & & \\
\hline Outpatient & $128(19.3)$ & & \\
\hline \multicolumn{4}{|c|}{ Sample acquisition, $n(\%)$} \\
\hline & All & Male & Female \\
\hline Indwelling catheter & $15(2.3)$ & 13 & 2 \\
\hline Disposable catheter & $148(23.2)$ & 2 & 146 \\
\hline Midstream urine & $476(74.5)$ & 464 & 12 \\
\hline \multicolumn{4}{|c|}{ Charlson comorbidity index } \\
\hline Mean \pm SD & $1.97 \pm 1.90$ & & \\
\hline
\end{tabular}

with hydrodynamic focusing in 2 different analysis chambers, the surface and core. Particles are stained by specific fluorochromes for nucleic acids and for surface structures and subsequently passed through the laser.

Counting and classification are based on signals of forwardscattered light, side-scattered light, and side-fluorescent light patterns. The pattern of individual light signals is transformed by specific algorithms into individual "fingerprints," allowing counting, identification, and classification into the particle categories. As compared to the previous cytometers of the UFC series, this model has significant technological innovations aimed at improving the sensitivity (SE) and specificity (SP) for the elements of the urinary sediment, particularly for the determination of bacteria.

\section{Culture-Based Diagnostics}

Bacterial growth analysis was performed routinely in the microbiological laboratory of the university hospital. Species identification was determined by MALDI-TOF analysis (bioMérieux) and supplemented using biochemical methods following MiQ standards [7].

\section{Decision Curve Analysis}

To estimate the clinical benefit of UFC as a diagnostic tool, we calculated a decision curve analysis following Vickers and Elkin [8].

\section{Results}

\section{Sample Population}

We analyzed a total of 662 urine samples from patients of a university department for urology; $75.2 \%$ were from male patients, and $24.8 \%$ were from female patients. A total 
Fig. 1. Microbiological spectrum as determined by urine culture and stratified into Gram-positive, Gram-negative, and Candida spp.

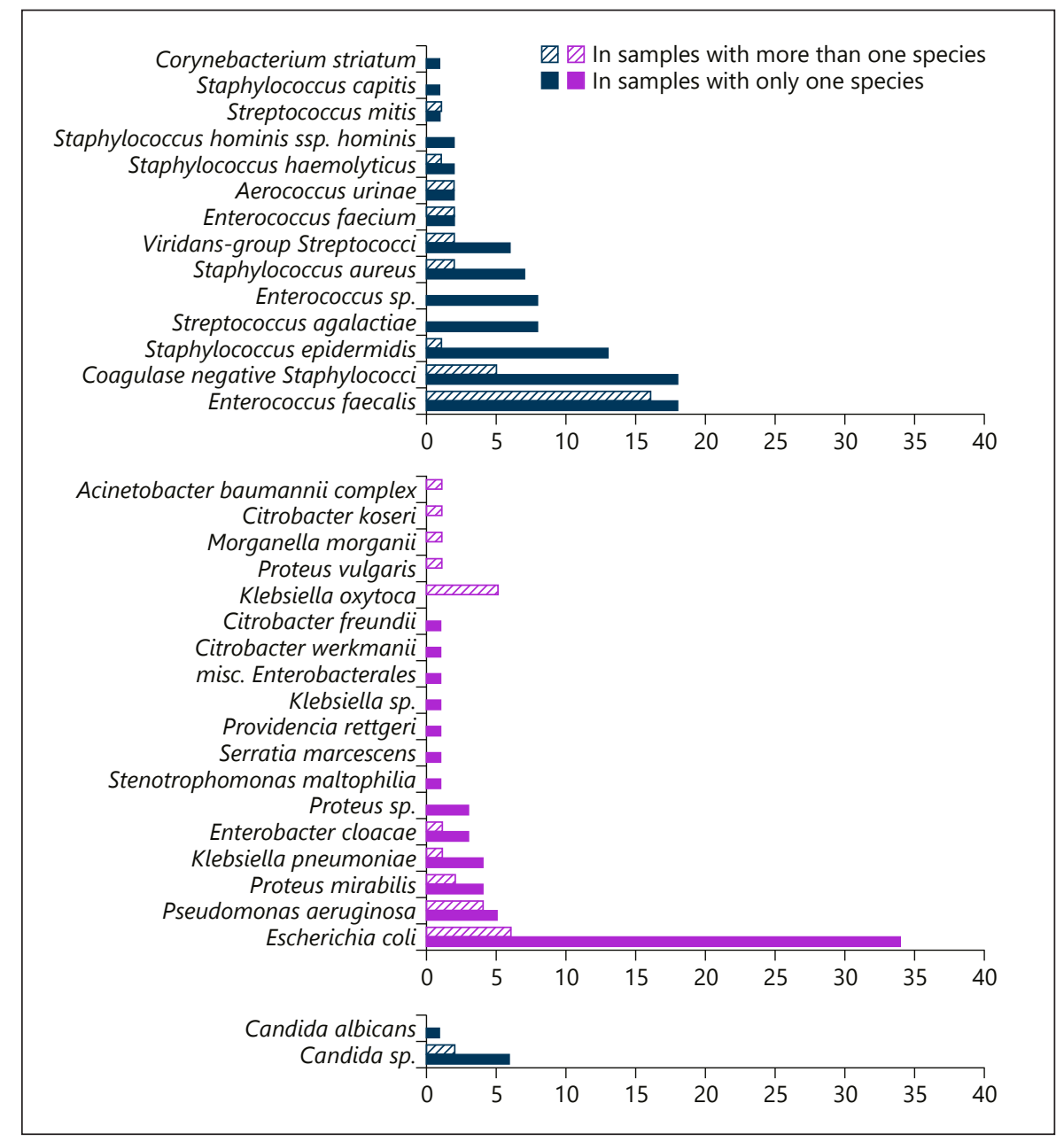

of $80.7 \%$ of the samples were from inpatients, and $19.3 \%$ were from outpatients. Following internal protocols, urine samples from female patients were taken via in-and-out catheters $(91.3 \%)$, whereas male patients provided midstream urine $(96.9 \%)$ if there was no catheter in place (3.1\%). The mean patient age was 58 years, and the mean Charlson comorbidity index was 1.97 (see Table 1) [9].

\section{Microbiological Spectrum}

In our samples, Escherichia coli was the most common uropathogen in samples with a single species. The second most common pathogen was Enterococcus faecalis, which was even found slightly more often in samples with 2 or more species. The third most common species were Staphylococcus epidermidis and other nonspecified coagulase-negative staphylococci. The remaining species included various Enterobacterales, other cocci, nonfermenters, and Candida spp. (see Fig. 1).

\section{Correlation between the Bacterial Inoculum in} Different Methods

We compared the bacterial inoculum as determined by UFC and classical culture methods. Figure 2 shows a significant correlation between the 2 methods (Pearson correlation coefficient 0.65 , $p$ value $<0.01 \%$ ).

The correlation analysis showed that UFC and culture are a better match when using high inoculums, a finding shared with other authors [10]. We investigated if they allow categorical agreement, specifically to determine whether "significant bacteriuria" is present. For this, we compared the data from both methods using 2 cutoffs, that is, in urine cultures of complicated UTI/pyelonephritis as specified by the EAU guidelines for urological infections, using $10^{5}$ colony-forming units $/ \mathrm{mL}(\mathrm{CFU} / \mathrm{mL})$ and $10^{4} \mathrm{CFU} / \mathrm{mL}$, respectively [1]. 


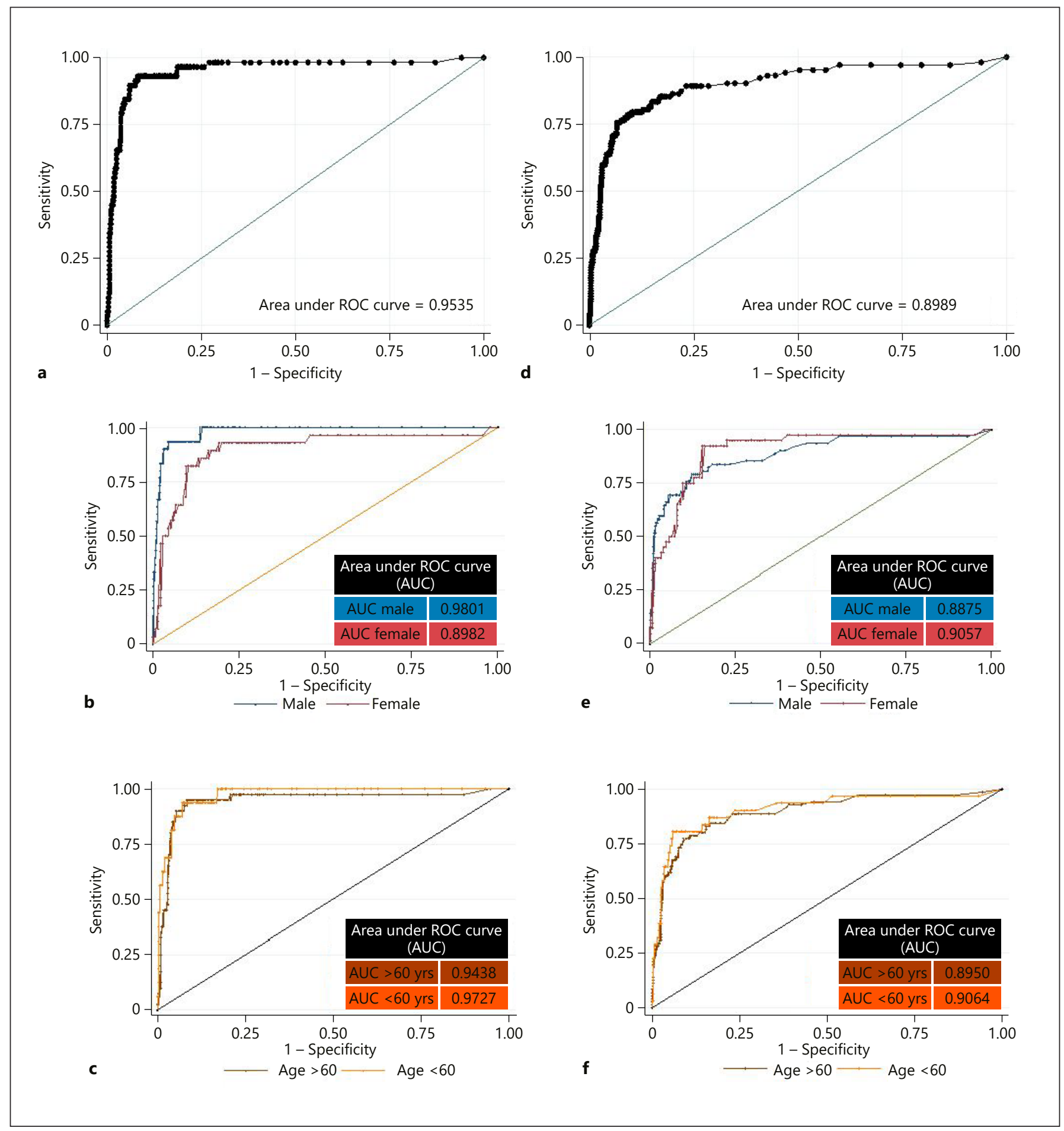

Fig. 2. ROCs for UFC to detect bacteriuria: detecting bacteriuria of $\geq 10^{5} \mathrm{CFU} / \mathrm{mL}$ with a UFC cutoff of $142 / \mu \mathrm{L}$. a Complete data set. b Samples analyzed separately by sex. c Samples analyzed separately by age. Detecting bacteriuria of $\geq 10^{4} \mathrm{CFU} / \mathrm{mL}$ with a UFC cutoff of $70 / \mu \mathrm{L}$. d Complete data set. e Samples analyzed separately by sex. f Samples analyzed separately by age. ROC, receiver operator characteristic; UFC, urine flow cytometry; CFU, colony-forming units; AUC, area under the receiver operating characteristic curve. 
Table 2. Accuracy of UFC to detect significant bacteriuria with subgroup analysis

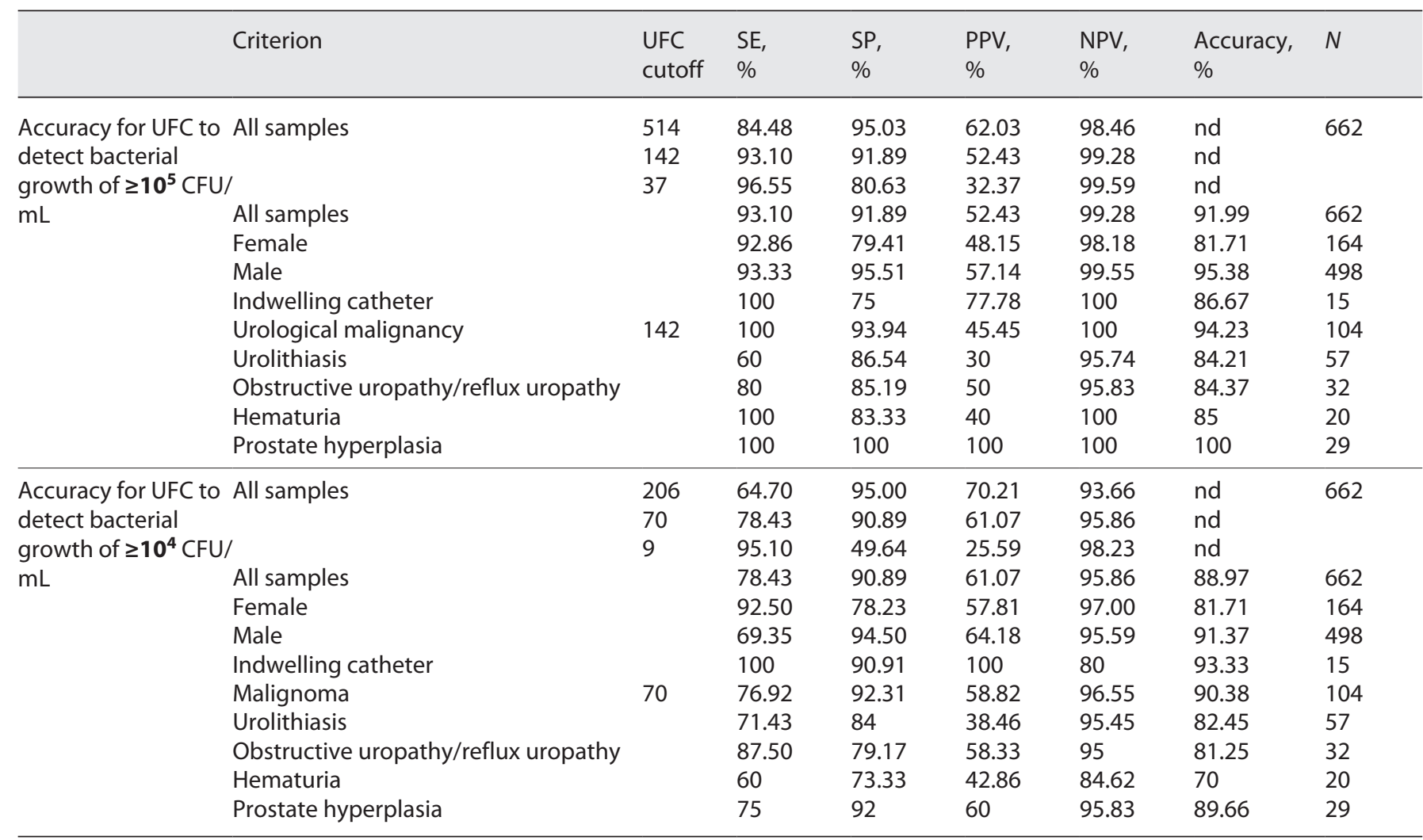

PPV, positive predictive value; NPV, negative predictive value; UFC, urine flow cytometry; CFU, colony-forming units; SE, sensitivity; SP, specificity.

\section{Analysis for Significant Bacteriuria of At Least $10^{5}$} CFU/mL

To determine the cutoff in UFC that could best distinguish between samples with at least $10^{5} \mathrm{CFU} / \mathrm{mL}$ and lower, we calculated receiver operating characteristics (ROCs). Youden analysis based on the ROC resulted in $142 / \mu \mathrm{L}$ bacteria measured by UFC as the best cutoff point to mirror $10^{5} \mathrm{CFU} / \mathrm{mL}$. We also determined ROCs separately for female and male patients and for patients younger and older than 60 years. We then calculated statistical contingency tables for various subgroups to evaluate as to whether significant bacteriuria would be missed in certain patient subgroups (see Fig. 2; Table 2).

False results: False-negative samples obtained using UFC consisted of 2 samples with $S$. epidermidis, 1 with $E$. faecalis, and 1 with both E. coli and Aerococcus urinae. Of the false-positive samples obtained, 30 were culturally sterile, 14 had growth between $10^{4}$ and $10^{5} \mathrm{CFU} / \mathrm{mL}, 3$ had growth $<10^{4} \mathrm{CFU} / \mathrm{mL}$, and 2 were mixed species.

\section{Analysis for Significant Bacteriuria of At Least $10^{4}$ $\mathrm{CFU} / \mathrm{mL}$}

We determined the urine UFC cutoff point to mirror bacteriuria of $\geq 10^{4} \mathrm{CFU} / \mathrm{mL}$. Youden analysis yielded 70/ $\mu \mathrm{L}$ as the optimal cutoff point for which we calculated statistical accuracy (see Fig. 2; Table 2).

False results with the above criteria: 22 samples were false negative by UFC, with inoculums of $10^{5} \mathrm{CFU} / \mathrm{mL}$ in 3 and $10^{4} \mathrm{CFU} / \mathrm{mL}$ in 19 samples (various species). Of the 51 false positive samples, 46 were sterile, 4 had growth between $10^{3}$ and $10^{4} \mathrm{CFU} / \mathrm{mL}$, and 1 had growth $<10^{3} \mathrm{CFU} / \mathrm{mL}$.

\section{Leukocyte Count by UFC versus Leukocyte Esterase by} Dipstick Testing

We compared the results of urine leukocyte esterase testing by the dipstick test system versus urine leukocyte counting by UFC. There was a significant correlation between leukocyte esterase and the leukocyte count in UFC (Pearson correlation coefficient 0.87 , $p$ value $<0.01 \%$, see online suppl. Fig. 1; for all online suppl. material, see 


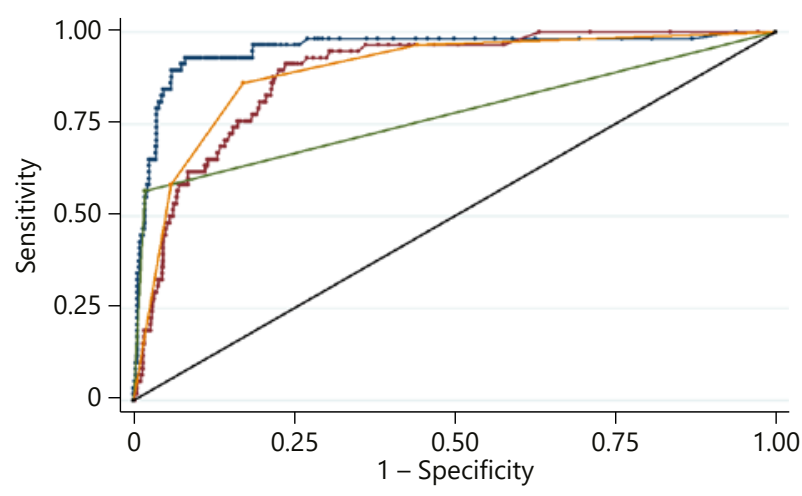

a

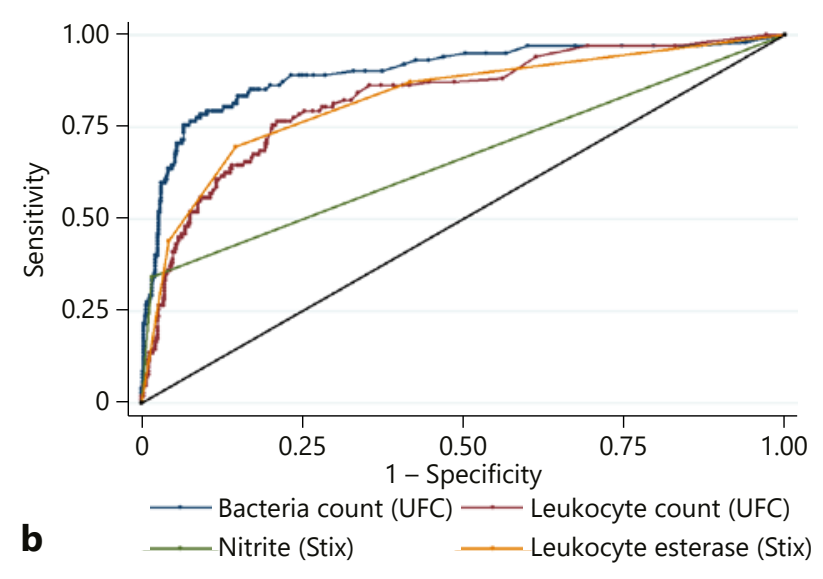

Fig. 3. ROCs comparing UFC and dipstick parameters to detect bacteriuria. a Cultural threshold of $\geq 10^{5} \mathrm{CFU} /$ $\mathrm{mL}$. b Cultural threshold of $\geq 10^{4} \mathrm{CFU} / \mathrm{mL}$. UFC, urine flow cytometry; CFU, colony-forming units; ROC, receiver operating characteristic.

www.karger.com/doi/10.1159/000520166), suggesting UFC as a suitable alternative method to leukocyte esterase testing for detecting leukocyturia.

\section{Comparison of Other Urine Cytometry Values to \\ Cultural Growth}

As leukocytes are often used as a surrogate parameter to detect UTIs, we plotted leukocytes number versus bacterial counts, both determined with UFC. The analysis showed a correlation (Pearson correlation coefficient 0.68 , $p$ value $<0.01 \%$ ) between leukocyte counts and bacterial counts, albeit with variance (see online suppl. Fig. 2).

For the more common uropathogens, we then determined how well bacterial counts, leukocyte counts, and erythrocyte counts correlated with bacterial growth in culture. As expected, erythrocytes had very little correlation with $E$. coli CFU numbers grown in sample cultures. Leukocyte numbers appear to increase with higher E. coli counts. However, E. coli culture counts were best reflected by bacterial counts using UFC. The same was true for the growth of E. faecalis. The results for Klebsiella were similar; however, there were fewer data points (see online suppl. Fig. 3).

\section{Decision Curve Analysis}

Decision curve analysis suggests UFC as a useful diagnostic tool for urine samples from urological patients (see Fig. 3).

Urine Flow Cytometry on Urological Samples

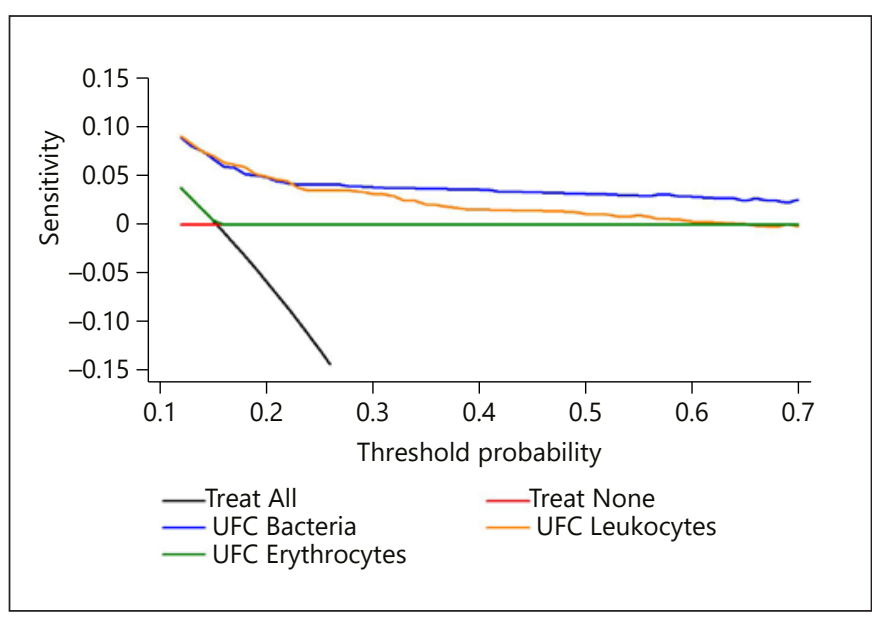

Fig. 4. Decision curve analysis for the net benefit of using UFC parameters for diagnosing bacteriuria. $x$ axis: threshold probability of UTI at which the patient would be classified as having UTI, and treatment would be initiated. $y$ axis: net benefit of diagnostic tool: proportion of true positive classifications subtracted by the proportion of false negatives weighted by the odds of the risk threshold. CFU, colony-forming units; UTI, urinary tract infections.

\section{Discussion}

The aim of our study was to evaluate UFC as a tool to screen patients for significant bacteriuria. Urological patients generally have additional comorbidities of the uro- 
Table 3. Combining UFC with dipstick parameters

\begin{tabular}{|c|c|c|c|c|c|c|}
\hline \multirow{4}{*}{$\begin{array}{l}\text { Accuracy and comparison of } \\
\text { UFC and Stix parameters to } \\
\text { detect bacterial growth of } \geq 10 \\
\text { CFU } / \mathrm{mL}\end{array}$} & Bacterial count (UFC) & 93.10 & 91.89 & 52.43 & 99.28 & 91.99 \\
\hline & Leukocyte count (UFC) & 91.38 & 76.16 & 26.90 & 98.92 & 77.49 \\
\hline & ${ }^{5}$ Leukocyte esterase (Stix) & 96.55 & 55.79 & 17.34 & 99.41 & 59.37 \\
\hline & Nitrite (Stix) & 56.90 & 98.18 & 75 & 95.95 & 94.56 \\
\hline \multirow{6}{*}{$\begin{array}{l}\text { Accuracy comparison of UFC } \\
\text { and Stix parameters to detect } \\
\text { bacterial growth of } \geq 10^{4} \text { CFU/ } \\
\mathrm{mL}\end{array}$} & Bacterial count (UFC) & 78.43 & 90.89 & 61.07 & 95.86 & 88.97 \\
\hline & Leukocyte count (UFC) & 76.71 & 78.89 & 39.59 & 94.84 & 78.40 \\
\hline & Leukocyte esterase (Stix) & 87.25 & 58.21 & 27.55 & 96.17 & 62.69 \\
\hline & Nitrite (Stix) & 34.31 & 98.39 & 79.55 & 89.16 & 88.52 \\
\hline & Leukocyte esterase + nitrite $(\text { Stix })^{1}$ & 88.24 & 58.21 & 27.78 & 96.45 & 62.84 \\
\hline & Composite testing ${ }^{2}$ & 90.20 & 56.07 & 27.22 & 96.91 & 61.33 \\
\hline
\end{tabular}

PPV, positive predictive value; NPV, negative predictive value; UFC, urine flow cytometry; CFU, colony-forming units; SE, sensitivity; SP, specificity. ${ }^{1}$ Sample considered positive "if either leukocyte esterase or nitrite was positive." "Sample considered positive "if either leukocyte esterase, nitrite, bacterial count (UFC), or leukocyte count (UFC) was positive."

genital tract, which could interfere with testing and impede results compared to those of a nonurological patient population [11]. Our collection of 662 samples has characteristics that might be particularly useful for urologists; the bacterial spectrum contains more enterococci and staphylococci than previously reported from nonurological patients. This result is of significance as some authors suspect UFC to be less reliable when detecting Gram-positive bacteria as compared to Gram-negative bacteria [12-16].

Our data show a clear correlation between UFC and culture (Fig. 4). To reach decisions regarding significant bacteriuria, cutoff points for the bacterial counts given by UFC are needed. Based on our data, we calculated UFC cutoffs for predicting significant bacteriuria in culture for 2 commonly used cutoffs, $10^{5} \mathrm{CFU} / \mathrm{mL}$ and $10^{4} \mathrm{CFU} / \mathrm{mL}$. Statistically, a cutoff of $142 / \mu \mathrm{L}$ worked best to determine bacteriuria of $\geq 10^{5} \mathrm{CFU} / \mathrm{mL}$, with a $99.28 \%$ negative predictive value (NPV), which would allow negative samples to be safely discarded. This cutoff had just a $52.43 \%$ positive predictive value (PPV) however with regards to the microbiological culture identifying $<10^{5} \mathrm{CFU} / \mathrm{mL}$. In the subgroup analysis, we noted that female samples were associated with a slight decrease in PPV (48.15\% vs. 57.14\%). These phenomena have been described by other authors for nonurological patients as well, suggesting systematic effects $[17,18]$. Indwelling bladder catheters did not seem to impact the measurements. With only 15 samples, this result is not truly conclusive but matches with one other studies that specifically investigated this component [19].
The NPV was good in patients with urological malignancy, but the PPV was slightly lower than that in the overall population ( $45.45 \%$ vs. $52.43 \%$ ). The PPV was even lower in the 57 samples from patients with urolithiasis $(30 \%)$. One could speculate that particles in the urine from patients with malignancies or stones might falsely be measured by UFC. We could however demonstrate that when compared to that in other patients, this result did not affect ruling out bacteriuria. Obstructive uropathy, hematuria, prostate hyperplasia, and age (patients younger or older than 60 years) did not compromise the accuracy of UFC.

Our comparison with the cultural cutoff of $\geq 10^{4} \mathrm{CFU} /$ $\mathrm{mL}$ yielded 70 bacteria/ $\mu \mathrm{L}$ as the optimal UFC cutoff, with an NPV of $95.86 \%$ and a PPV of $61.07 \%$. As mentioned before, samples from female patients were false positive more often (PPV 57.81\% vs. 64.18\%). Other authors found this as well, suggesting a systematic effect, possibly by virtue of the presence of fastidious bacteria such as lactobacilli commonly found in the female genital microbiota $[18,20,21]$.

As mentioned before, samples from patients with malignancy or urolithiasis were false positive more often (PPVs 58.82\% and 38.46\%), but NPVs were as high as those in the total population (95.45\% and $96.55 \%)$. Interestingly, the NPV was reduced in samples with hematuria $(84.62 \%)$. It is possible that UFC performs worse in samples with gross hematuria and low bacterial counts. Prostate hyperplasia $(n=32)$ and obstructive uropathy $(n=$ 
29) were not associated with such declines. While combining multiple parameters to rule out bacteriuria could be considered, in our calculations, no combination of dipstick parameters with or without UFC parameters allowed a better assessment than just bacterial count in UFC (Table 3).

Our data compare well with other studies $[5,17,19,22$, 23]. A meta-analysis by Shang et al. [5] with 19 studies comprising 22,305 samples calculates screening performance as follows: $0.92 \mathrm{SE}, 0.60 \mathrm{SP}$ for UTI screening when using bacterial counts, $0.87 \mathrm{SE}$, and $0.67 \mathrm{SP}$ when using leukocyte counts as surrogate. It seems problematic that data in this meta-analysis were pooled from studies using different reference standards $\left(>10^{3},>10^{4}\right.$ and $>10^{5} \mathrm{CFU} /$ $\mathrm{mL}$ ). Our data for $>10^{5} \mathrm{CFU} / \mathrm{mL}$ correspond well - reaching $0.93 \mathrm{SE}$ and $0.92 \mathrm{SP}$ - whereas data for the lower cutoff of $>10^{4} \mathrm{CFU} / \mathrm{mL}$ were less accurate, showing a SE of 0.78 and a SP of 0.91 . Notably, studies with lower statistical accuracy in the meta-analysis were among those that used lower cultural cutoffs values, suggesting that UFC is less reliable at the $>10^{4} \mathrm{CFU} / \mathrm{mL}$ threshold. The authors of the meta-analysis indicate that subjects enrolled were often not representative of clinical patient populations, a concern shared by urology experts regarding the specific urological population.

Methodologically, our study has the strength of being "double-blinded" since UFC/dipstick and culture analysis were done independently in different laboratories. All samples undergoing UFC were also investigated with culture methods. Other studies make exceptions for "contaminated" samples [5]. While species such as $S$. epidermidis may certainly be considered contaminants in many cases, they nevertheless are bacteria and should be counted when evaluating CFU, especially in patients with indwelling catheters.

A key difficulty in both evaluation and in the clinical use of UFC is that cutoff values are sought to make a binary decision (UTI yes/no) from a continuous parameter by setting arbitrary cutoff points. This approach creates problems specifically with samples where bacterial counts are close to these cutoff values. Müller et al. [24] developed a nomogram that tries to account for this [25]. We envision future cytometry reports that provide not only a simple " $\mathrm{n} / \mu \mathrm{L}$ " result but also include a depiction of the spectrum of expected cultural results, with their relative estimated probabilities. This approach would be incorporated in feedback algorithms in the laboratory software and could also include relevant patient information, such as sex or sample acquisition methods [11, $20,26]$.

Urine Flow Cytometry on Urological Samples
Our data suggest that UFC can be used to reliably rule out significant bacteriuria in urologic patients. In our laboratory, the analysis itself took about $5 \mathrm{~min}$ to perform (which amounts to a turnaround time from door-to-result of about an hour) and costs around 5 EUR. As it is more accurate than dipstick testing, we recommend its use.

Advantages of decision curve analysis include estimation of the clinical benefit of a testing strategy. In our example, it suggested a clear benefit for using UFC, particularly the use of the bacterial count parameter in comparison to the leukocyte parameter. A disadvantage of the decision curve analysis method is that in order to use it, one must beforehand decide on "threshold probabilities," that is, the reliability of a test a clinician might or might not be content with when using it. In our case however a net benefit could be calculated over the whole range of threshold probabilities, encouraging the usage of UFC despite its limitations.

In terms of antibiotic administration, urologists should be able to limit the use of antibiotic prescriptions for UTI when UFC results rule out significant bacteriuria and vice versa prescribe antibiotics when UFC suggests bacteriuria and therapy is clinically indicated. Microbiological culture might be reserved for urine samples with positive UFC results and samples with specific questions, in order to get resistance testing, thus saving resources.

A limitation of our study is the low sample size for subpopulations such as children, which may be important patients in centers serving pediatric populations. Nonurological studies in pediatric populations suggest cytometry to be viable for samples from children as well [27]. Likewise, future studies would benefit from additional samples from patients with specific diseases affecting the urogenital tract in order to enhance subgroup analysis. Technically, also UFC differentiation between Gram-positive cocci and Gram-negative rods in nextgeneration systems should be investigated.

We conclude that counting bacteria with UFC is an accurate and rapid method to determine significant bacteriuria in urological patients. In our analysis, it performed better than dipstick analysis or indirect parameters as leukocyturia and thus would make it the preferred choice.

\section{Acknlowledgments}

We thank Susanne Spesshardt, Martina Goldberg, and Christine Peschke for their excellent technical assistance. 


\section{Statement of Ethics}

This study was approved by the Ethics Committee of Justus Liebig University Giessen, Faculty of Medicine (AZ 80/17). Informed consent for this type of studies (improvement of diagnostic methods with unidentifiable patient data, on samples obtained within routine diagnostic, and without additional sample acquisition) was waived by the Ethics Committee of the Faculty of Medicine, Justus Liebig University Giessen (AZ 158/20).

\section{Conflict of Interest Statement}

F.W. declares personal fees and advisory board attendance and study participation from Achaogen, Bionorica, Klosterfrau HNealth Group, OM Pharma/Vifor Pharma, and Shionogi. He received personal fees for advisory board attendance from AstraZeneca, Janssen, LeoPharma, MerLion, MSD, Pfizer, RosenPharma, VenatoRx, and GSK. C.I. has served as a consultant to MSD Sharp \& Dohme and Shionogi. He has received lecture fees from MSD Sharp \& Dohme. The other authors have no conflicts of interest to declare.

\section{Funding Sources}

M.F. and M.O.G. both received grants from Deutsches Zentrum für Infektionsforschung (DZIF), which did not take part in the design or analysis of this study or in manuscript preparation.

\section{Author Contributions}

M.F., F.W., and B.A. designed the study. B.A. and H.R. acquired UFC data with help from M.G. M.G., M.F., C.I., and T.C. acquired microbiological culture data, and M.G. and F.W. acquired clinical urological data. M.G. and M.F. analyzed these data and made primary statistical analyses, which were reviewed and interpreted by all the authors. M.G. and M.F. drafted the manuscript, and all the authors revised and approved the submitted manuscript.

\section{Data Availability Statement}

All data generated or analyzed during this study are included in this article (and/or) its online supplementary material files. Further inquiries can be directed to the corresponding author.

\section{References}

1 EAU Guidelines. Edn. presented at the EAU Annual Congress Barcelona 2019. 2019 DOI: ISBN 978-94-92671-04-2.

2 Eisenhardt A, Schneider K, Hirche H, Lax H, Hadaschik B, Rehme C, et al. Diagnosis and empirical treatment of urinary tract infections in urologic outpatients. Urol Int. 2020; 104(7-8):617-24.

3 Humphries RM, Dien Bard J. Point-counterpoint: reflex cultures reduce laboratory workload and improve antimicrobial stewardship in patients suspected of having urinary tract infections. J Clin Microbiol. 2016 Feb;54(2): 254-8.

4 Monsen T, Ryden P. Erratum to: a new concept and a comprehensive evaluation of SYSMEX UF-1000i flow cytometer to identify culture-negative urine specimens in patients with UTI. Eur J Clin Microbiol Infect Dis. 2017;36(9):1705-3.

5 Shang YJ, Wang QQ, Zhang JR, Xu YL, Zhang WW, Chen Y, et al. Systematic review and meta-analysis of flow cytometry in urinary tract infection screening. Clin Chim Acta. 2013 Sep;424:90-5.

6 Paattiniemi EL, Karumaa S, Viita AM, Kärpänoja $P$, Mäkelä $M$, Isojärvi J, et al. Analysis of the costs for the laboratory of flow cytometry screening of urine samples before culture. Infect Dis. 2017 Mar;49(3):217-22.

7 Gatermann S, Fünfstück R, Handrick W, Leitritz L, Naber KG, Podbielski A, et al. MH. MIQ 02: Harnwegsinfektionen. 2nd ed. Amsterdam: Elsevier; 2005.
8 Vickers AJ, Elkin EB. Decision curve analysis: a novel method for evaluating prediction models. Med Decis Making. 2006 Nov;26(6): 565-74.

9 Quan H, Sundararajan V, Halfon P, Fong A, Burnand B, Luthi JC, et al. Coding algorithms for defining comorbidities in ICD-9-CM and ICD-10 administrative data. Med Care. 2005 Nov;43(11):1130-9.

10 Kim SY, Park Y, Kim H, Kim J, Koo SH, Kwon GC. Rapid screening of urinary tract infection and discrimination of gram-positive and gram-negative bacteria by automated flow cytometric analysis using Sysmex UF-5000. J Clin Microbiol. 2018;56(8).

11 Íñigo $\mathrm{M}$, Coello $\mathrm{A}$, Fernández-Rivas $\mathrm{G}$ Carrasco M, Marcó C, Fernández A, et al. Evaluation of the SediMax automated microscopy sediment analyzer and the Sysmex UF-1000i flow cytometer as screening tools to rule out negative urinary tract infections. Clin Chim Acta. 2016 Mar;456:31-5.

12 Manoni F, Valverde S, Antico F, Salvadego MM, Giacomini A, Gessoni G. Field evaluation of a second-generation cytometer UF100 in diagnosis of acute urinary tract infections in adult patients. Clin Microbiol Infect. 2002;8(10):662-8.

13 Manoni F, Fornasiero L, Ercolin M, Tinello A, Ferrian M, Hoffer P, et al. Cutoff values for bacteria and leukocytes for urine flow cytometer Sysmex UF-1000i in urinary tract infections. Diagn Microbiol Infect Dis. 2009 Oct; 65(2):103-7.
14 Zaman Z, Roggeman S, Verhaegen J. Unsatisfactory performance of flow cytometer UF100 and urine strips in predicting outcome of urine cultures. J Clin Microbiol. 2001;39(11): 4169-71.

15 Jiménez-Guerra G, Heras-Cañas V, ValeraArcas MD, Rodríguez-Grangér J, Navarro JM, Gutiérrez-Fernández J. Comparison between urine culture profile and morphology classification using fluorescence parameters of the Sysmex UF-1000i urine flow cytometer. J Appl Microbiol. 2017 Feb;122(2):473-80.

16 Yang CC, Yang SS, Hung HC, Chiang IN, Peng CH, Chang SJ. Rapid differentiation of cocci/mixed bacteria from rods in voided urine culture of women with uncomplicated urinary tract infections. J Clin Lab Anal. 2017 Sep;31(5):e22071.

17 De Rosa R, Grosso S, Lorenzi G, Bruschetta G, Camporese A. Evaluation of the new Sysmex UF-5000 fluorescence flow cytometry analyser for ruling out bacterial urinary tract infection and for prediction of Gram negative bacteria in urine cultures. Clin Chim Acta. 2018 Sep;484:171-8.

18 Sun SJ, Zuo LL, Liu PP, Wang XM, He ML, $\mathrm{Wu}$ SY. The diagnostic performance of urine flow cytometer UF1000i for urinary tract infections. Clin Lab. 2018 Sep 1;64(9):1395-401.

19 Le Z, Li F, Fei C, Ye A, Xie X, Zhang J. Performance of the Sysmex UF-1000i urine analyser in the rapid diagnosis of urinary tract infections in hospitalized patients. J Infect Chemother. 2016 Jun;22(6):377-82. 
20 Geerts N, Boonen KJ, Boer AK, Scharnhorst V. Cut-off values to rule out urinary tract infection should be gender-specific. Clin Chim Acta. 2016;452:173-6.

21 Kline KA, Lewis AL. Gram-positive uropathogens, polymicrobial urinary tract infection, and the emerging microbiota of the urinary tract. Microbiol Spectr. 2016;4(2).

22 de Boer FJ, Gieteling E, van Egmond-Kreileman H, Moshaver B, van der Leur SJ, Stegeman CA, et al. Accurate and fast urinalysis in febrile patients by flow cytometry. Infect Dis. 2017 May;49(5):380-7.
23 Moshaver B, de Boer F, van Egmond-Kreileman H, Kramer E, Stegeman C, Groeneveld P. Fast and accurate prediction of positive and negative urine cultures by flow cytometry. BMC Infect Dis. 2016;16:211.

24 Müller M, Seidenberg R, Schuh SK, Exadaktylos AK, Schechter CB, Leichtle AB, et al. The development and validation of different decision-making tools to predict urine culture growth out of urine flow cytometry parameter. PLoS One. 2018 Feb;13(2):e0193255.
25 Müller M, Seidenberg R, Schuh SK, et al. The development and validation of different decision-making tools to predict urine culture growth out of urine flow cytometry parameter. PLoS One. 2018;13(2):e0193255.

26 Mejuto P, Luengo M, Díaz-Gigante J. Automated flow cytometry: an alternative to urine culture in a routine clinical microbiology laboratory? Int J Microbiol. 2017;2017:8532736.

27 Conkar S, Mir S. Urine flow cytometry in the diagnosis of urinary tract infection. Indian J Pediatr. 2018 Nov;85(11):995-9. 